\title{
Bourgeonnement et synaptogenèse réactive dans l'épilepsie
}

Il existe dans l'épilepsie humaine et dans l'épilepsie expérimentale un remaniement des circuits dans le système nerveux central. Les modifications histologiques sont particulièrement nettes dans l'hippocampe, où l'on peut mettre en évidence un bourgeonnement des axones des cellules granulaires de la fascia dentata, les fibres moussues. Ce bourgeonnement et l'établissement de nouvelles synapses à caractère excitateur pourraient contribuer au maintien de l'épilepsie.

\section{Alfonso Represa Yehezkel Ben-Ari}

\section{ADRESSE}

A. Represa : chargé de recherches au Cnrs. Y. Ben-Ari : directeur de recherches au Cnrs. Inserm U. 29, 123, boulevard Port-Royal,

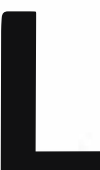

'activation répétée d'un circuit neuronal se traduit souvent par une modification persistante de l'efficacité synaptique. Ainsi, l'application d'un train de stimulations électriques de haute fréquence produit, au niveau des structures corticales comme l'hippocampe, une potentialisation à long terme de la transmission synaptique, qui est considérée à l'heure actuelle comme un modèle expérimental de mémoire et de plasticité synaptique. Par de nombreux travaux, on a essayé d'établir si la quasi-permanence de la potentialisation était due à des modifications morphologiques, notamment à la formation de nouvelles synapses. Dans ce travail, nous décrirons des résultats récents qui suggèrent qu'une hyperactivité neuronale de brève durée, comme celle qui accompagne des crises épileptiques, induit un bourgeonnement des fibres moussues de l'hippocampe et la formation de synapses aberrantes de type excitateur. Outre son intérêt pour la compréhension de l'épilepsie, cette néosynaptogenèse constitue un excellent modèle expérimental d'étude de la synaptogenèse et de la plasticité.

\section{La formation hippocampique et les récepteurs au glutamate}

Structure corticale simplifiée, organisée de façon laminaire, la formation hippocampique est composée schématiquement de trois types de neurones : les cellules granulaires, les cellules pyramidales et les interneurones. Les cellules granulaires et les cellules pyramidales sont organisées en deux couches facilement identifiables qui adoptent la forme de deux croissants qui s'emboîtent (figure $1 B$, p. 878). Les interneurones, en revanche, apparaissent dispersés. La propagation de l'influx nerveux se fait 
le long d'un réseau trisynaptique composé successivement : 1) des axones du faisceau perforant, principale afférence extrinsèque de l'hippocampe, provenant du cortex entorhinal et qui innerve les dendrites apicales des cellules granulaires ; 2) des fibres moussues, provenant des cellules granulaires, innervant les dendrites apicales proximales des cellules pyramidales de la région CA 3 ; 3) et finalement des axones des cellules pyramidales de CA3 (collatérales de Schaffer) qui vont innerver les cellules pyramidales de la région CA1.

Le médiateur de ces trois synapses excitatrices est vraisemblablement l'acide glutamique [1]. L'acide glutamique agit par l'intermédiaire de deux grands types de récepteurs caractérisés par leur sensibilité sélective aux agonistes et antagonistes, leur voltage-dépendance et leur différente distribution régionale : 1) les récepteurs de type NMDA (Nméthyl-D-aspartate) sont associés à un canal ionique perméable au $\mathrm{Ca}^{2+}$; 2) les récepteurs de type QUIS (quisqualate)/KA (kaïnate) sont associés à des canaux perméables aux cations monovalents $\mathrm{Na}^{+}$ et $\mathrm{K}^{+}[2]$.

\section{Propriétés particulières des fibres moussues}

La synapse entre la fibre moussue et la cellule pyramidale de CA3 est un complexe synaptique très caractéristique. Le bouton présynaptique est géant (jusqu'à 6 à $8 \mu \mathrm{m}$ de diamètre), il a une forme complexe, avec de multiples replis et contient de très nombreuses vésicules rondes. Le bouton terminal de la fibre moussue est riche en zinc, ce qui permet de le colorer de façon relativement sélective à l'aide de la méthode de Timm (figure 2, p. 880). Il établit plusieurs contacts synaptiques asymétriques avec les épines de la partie proximale des dendrites de CA3.

L'étude par autoradiographie sur coupes de la distribution des différents types de récepteurs aux acides aminés excitateurs permet d'identifier les fibres moussues. En effet, si la densité des sites de liaison de type NMDA est particulièrement élevée au niveau des couches dendritiques de CA1 (figure 1C) ou de CA3, les $\mathrm{m} / \mathrm{s} n^{\circ} 9$, vol. 6 , novembre 90 sites de liaison de haute affinité à l'acide kaïnique sont particulièrement enrichis dans la zone de terminaison des fibres moussues, le stratum lucidum de CA3 (figure 1D). Des études autoradiographiques après lésion sélective des éléments pré- ou postsynaptiques suggèrent que les sites de liaison au $\mathrm{KA}$ sont préférentiellement localisés (plus de $50 \%$ ) en position présynaptique sur les terminaisons des fibres moussues où ils moduleraient la libération de glutamate et d'aspartate [3]. Vingt pour cent seulement de ces sites pourraient être localisés, post synaptiquement, sur les neurones pyramidaux de CA3 [4]. Les sites de liaison au KA sont très résistants aux conditions de dégradation, ce qui permet d'appliquer cette technique à des tissus humains prélevés en postmortem [5]. Les études de la maturation des sites de liaison à l'acide kaïnique chez le rat [6] montrent aussi une très bonne corrélation avec la maturation de la synapse moussue. Ces résultats suggèrent que les sites de liaison à l'acide kaïnique peuvent être considérés comme un marqueur spécifique de la synapse moussue ; l'étude autoradiographique de ces sites peut ainsi être utilisée pour suivre, de façon quantitative, les modifications de fibres moussues chez les sujets épileptiques, et notamment chez l'homme, où l'analyse en microscopie électronique, ainsi que les colorations histochimiques ne sont pas sans poser quelques difficultés techniques.

La région CA 3 de l'hippocampe est la structure la plus épileptogénique du cerveau ; plusieurs données expérimentales suggèrent que les fibres moussues jouent un rôle important dans l'origine et le contrôle des décharges épileptiformes et dans les séquelles histopathologiques associées (la sclérose de la corne d'Ammon). La destruction des fibres moussues réduit en effet les crises épileptiques induites par le KA de même que la dégénérescence de la région CA3 qui lui est associée [7].

\section{Bourgeonnement des fibres moussues dans I'épilepsie expérimentale}

Dans le kindling ou phénomène d'embrasement, les crises convulsives sont provoquées par des stimulations électriques quotidiennes des différentes structures appartenant au système limbique. Les animaux ainsi traités présentent, par la suite, un état d'hyperexcitabilité permanente ainsi qu'une vulnérabilité accrue aux agents convulsivants. Le kindling se caractérise, d'un point de vue électrographique, par: l'augmentation progressive de la durée des postdécharges induites par la stimulation délivrée ; la propagation des postdécharges vers d'autres structures cérébrales et l'abaissement durable du seuil d'obtention de ces postdécharges. D'un point de vue clinique-électrographique, le kindling se rapproche de l'épilepsie du lobe temporal chez l'homme. Cependant, dans ce modèle d'épilepsie, aucune lésion n'est connue [8].

Les données histochimiques et autoradiographiques montrent clairement, après kindling chez le rat, un bourgeonnement des fibres moussues de l'hippocampe, qui forment une bande aberrante au niveau de la couche supragranulaire [8, 9]. La figure 2 montre que les fibres moussues (ici marquées par la coloration de Timm et le $\left.\left[{ }^{3} \mathrm{H}\right] \mathrm{KA}\right)$ forment aussi une bande aberrante au niveau de la région CA3, dans la couche infrapyramidale (stratum oriens).

Des données similaires ont été observées avec un autre modèle d'épilepsie du lobe temporal provoqué celuici, par l'administration intrapéritonéale ou intracérébrale d'acide kaïnique [10]. Cette procédure induit un état de mal épileptique, suivi du développement d'une nécrose des cellules hippocampiques, notamment de la région CA3-CA4; cette lésion est semblable à la sclérose hippocampique observée chez des sujets ayant une épilepsie du lobe temporal. Plusieurs travaux ont montré que les rats traités à l'acide kaïnique présentent un bourgeonnement des fibres moussues au niveau supragranulaire $[4,11]$.

Des études en microscopie électronique confirment, par ailleurs, la formation de nouvelles synapses dans les régions innervées de façon aberrante. Ainsi, après kindling on observe dans la région supragranulaire [8] et dans la région infrapyramidale de CA3 (A. 


\section{RÉFÉRENCES}

1. Cotman CW, Iversen LL. Excitatory amino acids in the brain-focus on NMDA receptors. Trends Neurosci 1987; 10 : 263-302.

2. Monaghan DT, Bridges RJ, Cotman CW. The excitatory amino acid receptors : their classes, pharmacology, and distinct properties in the function of the central nervous system. Ann Rev Pharmacol Toxicol $1989 ; 29: 365-402$.

3. Ferkany JW, Zaczek R, Coyle JT. Kainic acid stimulates excitatory amino acid neurotransmitter release at presynaptic receptor. Nature 1982 ; 298 : 279-82.

4. Represa A, Tremblay E, Ben-Ari Y. Kainate binding sites in the hippocampal mossy fibers : localization and plasticity. Neuroscience $1987 ; 20: 739-48$.

5. Tremblay E, Represa A, Ben-Ari Y. Autoradiographic localization of kainic acid binding sites in the human hippocampus. Brain Res 1985 ; 343 : 378-82.

6. Represa A, Tremblay F, Schoevart D, Ben-Ari Y. Development of high affinity kainate binding sites in human and rat hippocampi. Brain Res 1986 ; 384 : 170-4. 7. Okazaki MM, Nadler JV. Protective effects of mossy fiber lesions against kainic acid-induced seizures and neuronal degeneration. Neuroscience $1988 ; 26: 763-81$.

8. Sutula T, Xiao-Xian H, Cavazos J, Scott G. Synaptic reorganization in the hippocampus induced by abnormal functional activity. Science $1988: 239: 1147-50$.

9. Represa A, Le Gall La Salle G, BenAri Y. Hippocampal plasticity in the kindling model of epilepsy in rats. Neurosci Lett $1989 ; 99$ : 345-50.

10. Ben-Ari Y. Limbic seizures and brain damage produced by kainic acid : mechanisms and relevance to human temporal lobe epilepsy. Neuroscience $1985 ; 14$ : 375-403.

11. Frotscher M, Zimmer J. Lesion induced mossy fibers to the molecular layer of the rat fascia dentata : identification of postsynaptic granule cells by the Golgi-EM technique. J Comp Neurol 1983 ; $215: 299-311$. 12. Represa A, Robain O, Tremblay E, Ben-Ari Y. Hippocampal plasticity in childhood epilepsy. Neurosci Lett 1989b; 99 : 351-5.

13. Lanerolle NC, Kim JH, Robbins RJ, Spencer DD. Hippocampal interneuron loss and plasticity in human temporal lobe epi-
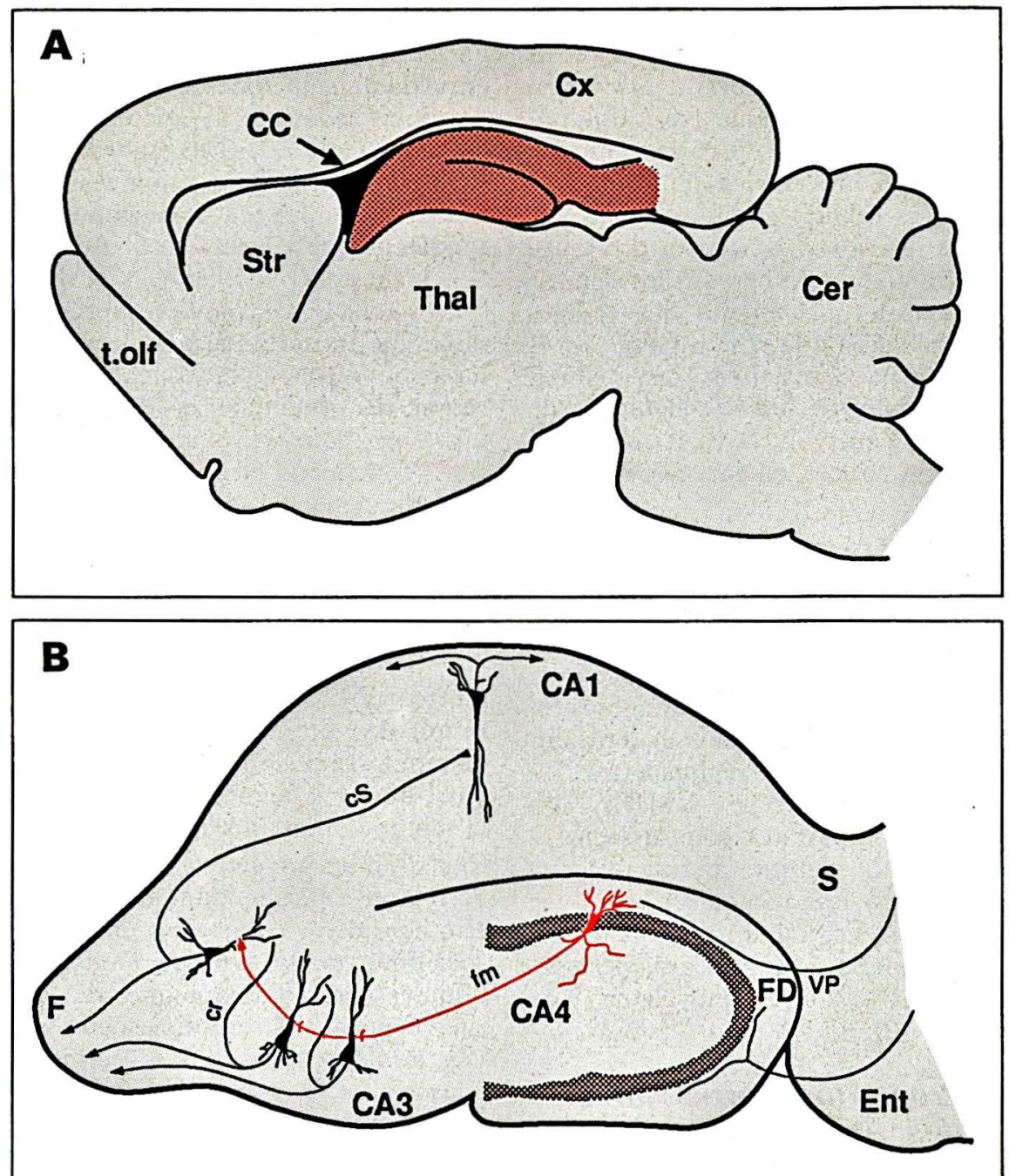

Figure 1. (A) Schéma d'une coupe sagittale de l'encéphale montrant la localisation de la formation hippocampique (en pointillés). (B) Représentation de l'organisation synaptique basique de la formation hippocampique : elle débute au niveau de la fascia dentata (FD), qui constitue la principale zone d'arrivée des afférences du cortex entorhinal (Ent); les axones des cellules granulaires de la fascia dentata (les fibres moussues; fm) innervent la région CA3 ; les collatérales des axones des cellules pyramidales de CA3 (collatérales de Schaffer; cS) innervent à leur tour les cellules pyramidales de CA 1 ; finalement, les cellules de CA1 et CA3 innervent la région subiculaire (S). Les axones des cellules pyramidales utilisent la fimbria (F) pour atteindre d'autres structures. En pointillés, la couche granulaire de la fascia dentata. En gris le stratum pyramidal de la corne $d^{\prime}$ Ammon et du subiculum. 

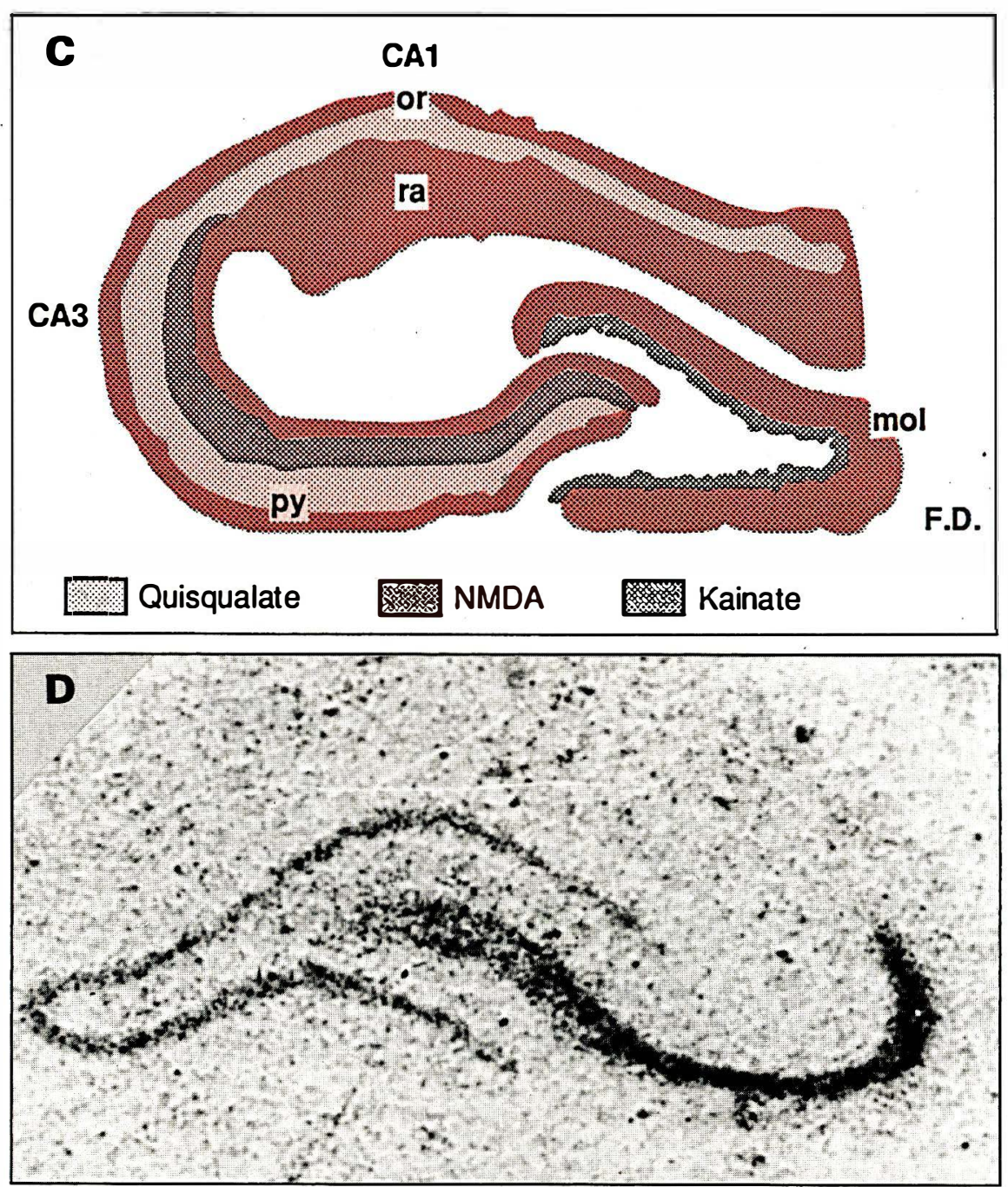

Figure 1. (C) Distribution, au niveau de la formation de I'hippocampe, des trois principaux agonistes des acides aminés excitateurs. Les zones de terminaison de la voie entorhinale (stratum moléculaire) et des collatérales de Schaffer (stratum radiatum) sont particulièrement enrichies en sites de liaison au NMDA. Le stratum lucidum et la couche supragranulaire présentent les plus fortes densités de sites de liaison au kaïnate. (D) Autoradiographie montrant la distribution des sites de liaison au kaïnate dans la formation hippocampique chez le rat. $C C$ : corpus callosum ; Cer : cerebellum ; $C x$ : néocortex; cS : collatérale de Schaffer ; cr : collatérale récurrente ; Ent : cortex entorhinal ; $F$ : fimbria ; FD : fascia dentata ; fm : fibre moussue; mol : couche moléculaire ; or: stratum oriens ; py : stratum pyramidal ; ra : stratum radiatum ; Str : striatum ; T. olf : tubercule olfactif ; Thal : thalamus ; $v p$ : voie entorhinale; $S$ : subiculum.
Represa, O. Robain et Y. Ben-Ari en préparation) des boutons synaptiques présentant les caractéristiques des boutons synaptiques moussus (boutons synaptiques géants, multiinvaginés, établissant des contacts synaptiques multiples, de type asymétrique avec les épines dendritiques). La figure 3 ( $p$. 881) montre, en particulier, des synapses moussues typiques au niveau proximal des dendrites basilaires des cellules pyramidales de CA3. Il en est de même chez les animaux traités au KA. En effet, des études effectuées par microscopie électronique combinée à la coloration de Golgi [11] montrent une augmentation du nombre de boutons synaptiques moussus au niveau de la fascia dentata des animaux traités au kaïnate ; ces boutons assurent des contacts synaptiques asymétriques avec des épines dendritiques des cellules granulaires de la fascia dentata.

\section{Bourgeonnement des fibres moussues chez le sujet épileptique}

Nous avons effectué une étude histologique et autoradiographique sur des hippocampes de 7 enfants qui avaient présenté des crises d'épilepsie, sévères, d'étiologie diverse [12]. Les résultats ont été comparés à ceux obtenus sur 8 sujets d'âges semblables, décédés sans avoir eu d'altérations neurologiques. La coloration de Timm et l'autoradiographie des sites de liaison au KA (figure 4, p. 882) montrent une augmentation significative du marquage caractéristique des fibres moussues dans les régions normalement innervées, ainsi que la présence de fibres moussues aberrantes au niveau supragranulaire et infrapyramidal, accompagnée d'une augmentation très significative de la densité des sites de liaison au KA, sans que leur constante d'affinité ne soit affectée. D'autres études, effectuées sur des hippocampes prélevés chirurgicalement sur des sujets adultes atteints d'épilepsie incurable du lobe temporal, montrent des résultats similaires [13-15].

\section{Les nouvelles synapses moussues sont-elles fonctionnelles?}

Une seule étude électrophysiologique $\overline{879}$ 


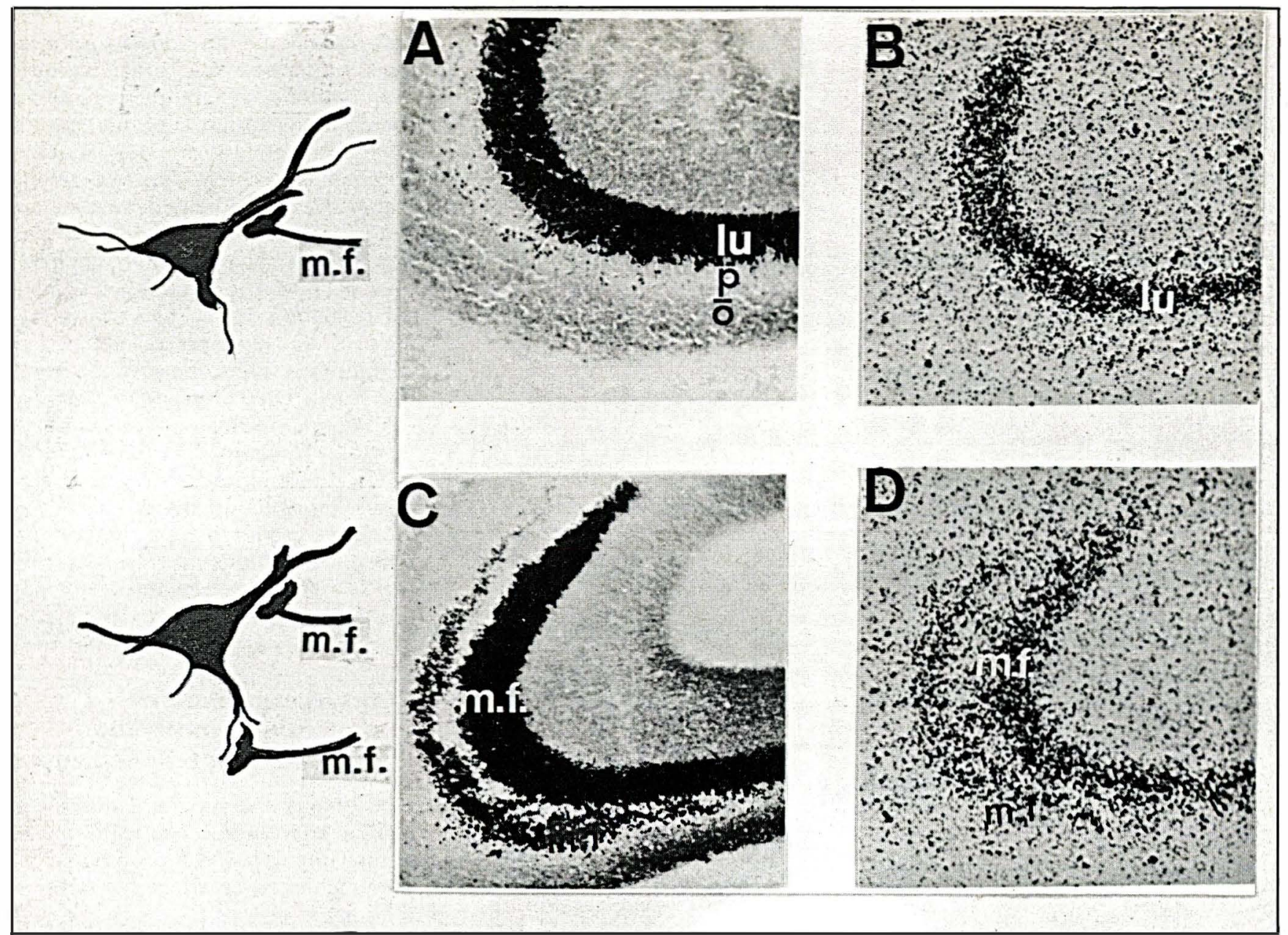

Figure 2. Bourgeonnement des fibres moussues après le kindling chez le rat. Tandis que chez le rat contrôle (A et $B$ ), les fibres moussues forment une seule bande suprapyramidale (stratum lucidum) au niveau de la région CA3, chez les animaux épileptiques, elles forment deux bandes; ainsi les cellules pyramidales de CA3 apparaissent doublement innervées par les fibres moussues : au niveau des dendrites apicales (stratum lucidum) et des dendrites basilaires (stratum oriens ou infrapyramidal). A et $C$ : coloration des fibres moussues par la méthode de Timm; $B$ et $D$ : autoradiographies des sites de liaison au KA, qui est considéré comme un marqueur sélectif des fibres moussues (m.f.). Lu, $P$ et $O$ : stratum lucidum, pyramidal et oriens respectivement. (modifié $d^{\prime}$ après [31]).

portant sur les effets de cette réinnervation a été effectuée jusqu'à présent [16] ; elle suggère que les fibres moussues récurrentes forment en fait un circuit de caractère excitateur. En effet, sur des tranches d'hippocampe d'animaux traités au kaïnate, la stimulation antidromique ${ }^{*}$ des fibres moussues au niveau de la région CA4, induit au niveau des cellules granulaires, des bouffées de potentiels d'action, alors qu'un seul potentiel d'action est observé chez les animaux témoins. En revanche, si l'expérience est réalisée avec un milieu contenant une forte concentration de magnésium et pas de calcium (ce qui permet de bloquer totalement la trans- mission synaptique), la même stimulation antidromique n'induit pas de bouffées de potentiels d'action, mais le simple potentiel d'action observé chez les témoins. Cela suggère que ces bouffées de potentiels d'action pourraient être liées à l'existence de synapses excitatrices formées à partir du circuit récurrent des fibres moussues.

En outre, l'application de bicuculine (un antagoniste des récepteurs GABA A) sur des tranches témoins bloque l'apparition des bouffées de potentiels d'action après stimulation antidromi-

\footnotetext{
- Antidromique : dans le sens axone $\rightarrow$ corps cellulaire, c'est-a-dire inverse de celui de la transmission de l'influx nerveux.
}

que des fibres moussues. Ainsi ces bouffées ne seraient pas liées à une désinhibition des synapses déjà existantes, mais bien à la formation de nouvelles synapses aberrantes.

Bien que cette étude, effectuée à l'aide d'enregistrements extracellulaires, ne permette pas de décrire les effets de cette néosynaptogenèse sur l'excitabilité des cellules, elle suggère néanmoins que le circuit aberrant cellule granulaire-cellule granulaire est fonctionnel. On peut raisonnablement penser que l'hyperinnervation moussue va jouer un rôle dans l'hyperexcitabilité hippocampique, notamment par l'augmentation de la libération de zinc et la densité des 
sites de liaison à l'acide kaïnique. En effet, le zinc - qui est particulièrement concentré dans les boutons synaptiques des fibres moussues et libéré par stimulation [17] - réduit : (1) l'inhibition GABAergique ; (2) les conductances $\mathrm{K}^{+}$dépendantes du calcium, qui repolarisent la cellule après une bouffée de potentiels [18]. Une augmentation des taux de zinc induirait par conséquent une augmentation de l'excitabilité des cellules pyramidales de CA3. L'augmentation de la densité des récepteurs au KA va également augmenter l'excitabilité hippocampique, puisque, sur des tranches d'hippocampe [19] comme in vivo, l'application de très faibles concentrations de $\mathrm{KA}$ (20-50 nM) produit au niveau des cellules pyramidales de CA3 des activités paroxystiques. Celles-ci sont dues à l'action dépolarisante du KA produite par l'activation d'une conductance cationique et également à la réduction des conductances potassiques dépendantes du calcium $\left(\mathrm{I}_{\mathrm{Q}}\right.$ et $I_{A H P}$ respectivement) [19] et des courants GABAergiques [20]. L'augmentation de la densité des sites de liaison à l'acide kaïnique, particulièrement dans la région CA3, pourrait être l'un des éléments impliqués dans le maintien de l'hyperexcitabilité.

Des données préliminaires suggèrent d'ailleurs qu'il y a une corrélation significative entre la densité des fibres moussues aberrantes au niveau supragranulaire et la fréquence des épisodes convulsifs spontanés observés chez des rats traités au KA [21]. Bien que cette corrélation ne concerne qu'une partie des animaux observés, il semblerait que les animaux qui présentent des convulsions spontanées aient un degré de bourgeonnement beaucoup plus important que ceux ne présentant pas de convulsions spontanées.

\section{Quels sont les mécanismes responsables de ce bourgeonnement?}

On sait que des lésions ou des dénervations partielles de l'hippocampe (notamment la lésion des voies afférentes cholinergiques et entorhinales) induisent un bourgeonnement réactif, notamment des fibres moussues. Le $m / s n^{\circ} 9$, vol. 6 , novembre 90

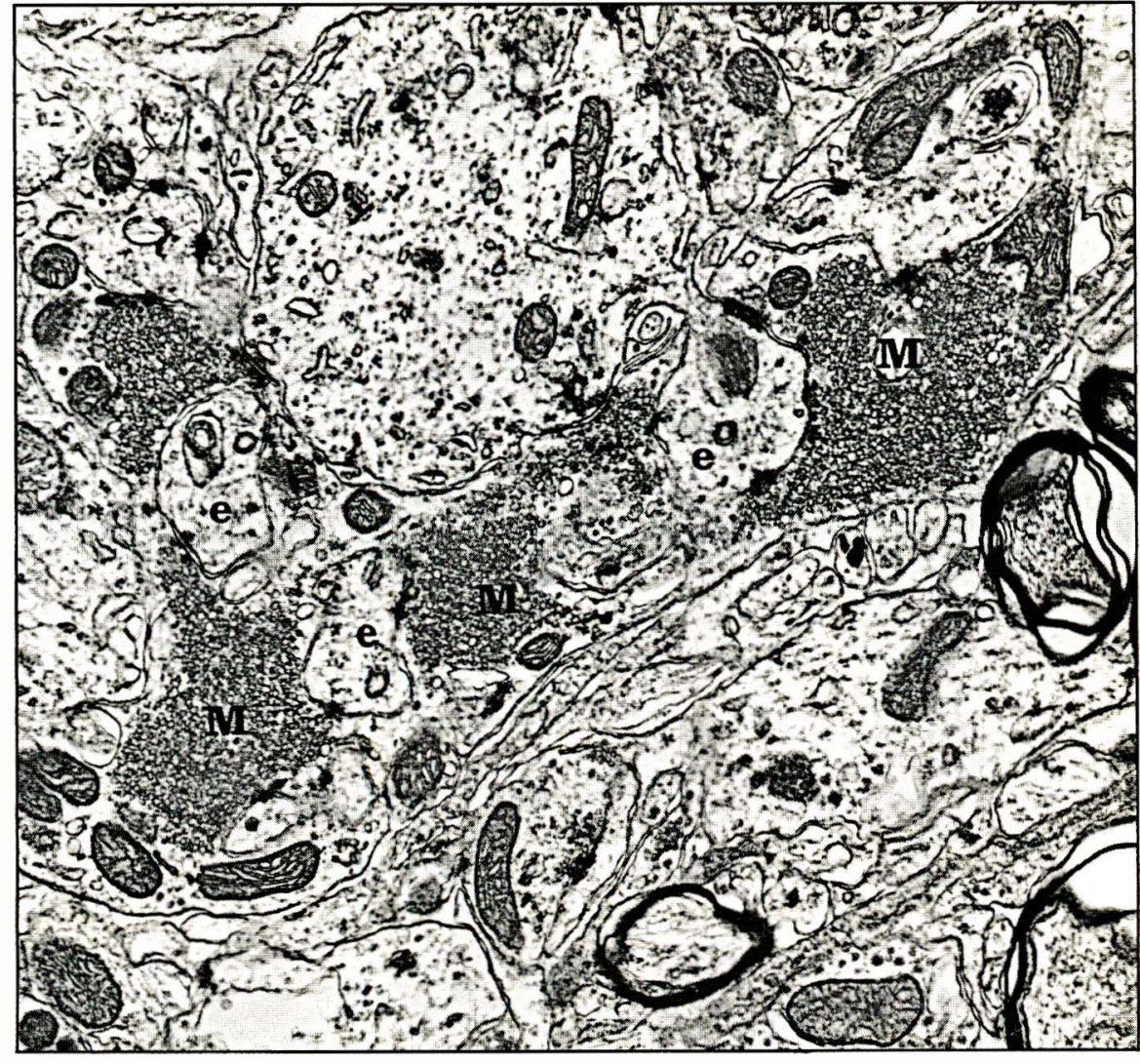

Figure 3. Image en microscopie électronique de la couche infrapyramidale de CA3 d'un rat soumis au kindling; des boutons synaptiques moussus ectopiques (M) font des synapses asymétriques avec des épines dendritiques (e) (x8900).

bourgeonnement des fibres moussues observé après traitement au KA pourrait être dû aux lésions qui accompagnent les états convulsifs. Cependant, il est clair que chez des animaux soumis au kindling, il y a un bourgeonnement de ces fibres en l'absence de lésions. En outre, le bourgeonnement des fibres moussues a été observé dans les stades précoces du kindling, avant même l'apparition des décharges convulsives associées à des manifestations motrices [8]. Par conséquent, il est possible que l'hyperexcitabilité soit capable d'induire chez l'adulte un bourgeonnement des fibres moussues et une néosynaptogenèse en l'absence des lésions.

On a pu récemment identifier un certain nombre d'étapes intermédiai- res entre l'hyperactivité transitoire initiale et le bourgeonnement réactif.

Une brève crise paroxystique produit, dans des tranches d'hippocampe, une modification à long terme de la réponse synaptique ; cette modification est caractérisée par le fait qu'après une crise brève, les cellules pyramidales de CA3 répondent par une décharge épileptiforme à la stimulation des fibres moussues ou d'autres afférences de la région CA3, au lieu du potentiel postsynaptique excitateur évoqué avant la crise par les mêmes stimulations [19]. Cet effet n'est pas sans rappeler la potentialisation à long terme, puisqu'il est bloqué par des antagonistes des récepteurs au NMDA [19]. Il est clair par conséquent, que l'épileptogenèse est associée à une entrée cellulaire de 
14. Sutula T, Cascino G, Cavazos J, Parada I, Ramirez L. Mossy fiber synaptic reorganization in the epileptic human temporal lobe. Ann Neurol 1989; 26 : 321-30.

15. Houser CR, Miyashiro JE, Swartz BE, Walsh GO, Rich JR, Delgado-Escueta AV. Altered patterns of dynorphin immunoreactivity suggest mossy fiber reorganization in human hippocampal epilepsy. Neuroscience 1990 ; in press.

16. Tauck DL, Nadler JV. Evidence for functional mossy fiber sprouting in hippocampal formation of kainic acid treated rats. $J$ Neurosci 1985 ; 5 : 1016-22.

17. Aniksztejn L, Charton G, Ben-Ari Y. Selective release of endogenous zinc from the hippocampal mossy fibers in situ. Brain Res 1987 ; 404 : 58-64.

18. Sim JA, Cherubini E. Submicromolar concentration of zinc irreversibly reduces a calcium-dependent potassium current in rat hippocampal neurones in vitro. Neuroscience 1990 ; sous presse.

19. Ben-Ari Y, Gho M. Long-lasting modification of the synaptic properties of rat CA3 hippocampal neurones induced by kainic acid. J Physiol 1988 ; 404 : 365-84.

20. Rovira C, Gho M, Ben-Ari Y. Block of GABAb-activated $\mathrm{K}^{+}$conductance by kainate and quisqualate in rat CA3 hippocampal pyramidal neurones. Eur J Physiol $1990 ; 415: 471-8$

21. Cronin J, Dudek FE. Chronic seizures and collateral sprouting of dentate mossy fibers after kainic acid treatment in rats. Brain Res 1988 ; 474: 181-4.

22. Malenka RC, Kauer JA, Perkel DJ, Nicoll RA. The impact of postsynaptic calcium on synaptic transmission-its role in long term potentiation. Trends Neurosci $1989 ; 12$ : 444-50.

23. Dragunow M, Robertson HA. Kindling stimulation induces c-fos protein(s) in granule cells of the rat dentate gyrus. Nature $1987 \mathrm{~b} ; 329$ : 441-2.

24. Popovici Th, Barbin G, Ben-Ari Y. Kainic acid induced seizures increase c-foslike protein in the hippocampus. Eur J Pharmacol $1988 ; 150$ : 405-6.

25. Le Gal La Salle G. Long-lasting sequential increase of $\mathrm{c}$-fos oncoprotein expression in kainic acid-induced status epilepticus. Neurosci Lett $1988 ; 88: 127-30$.
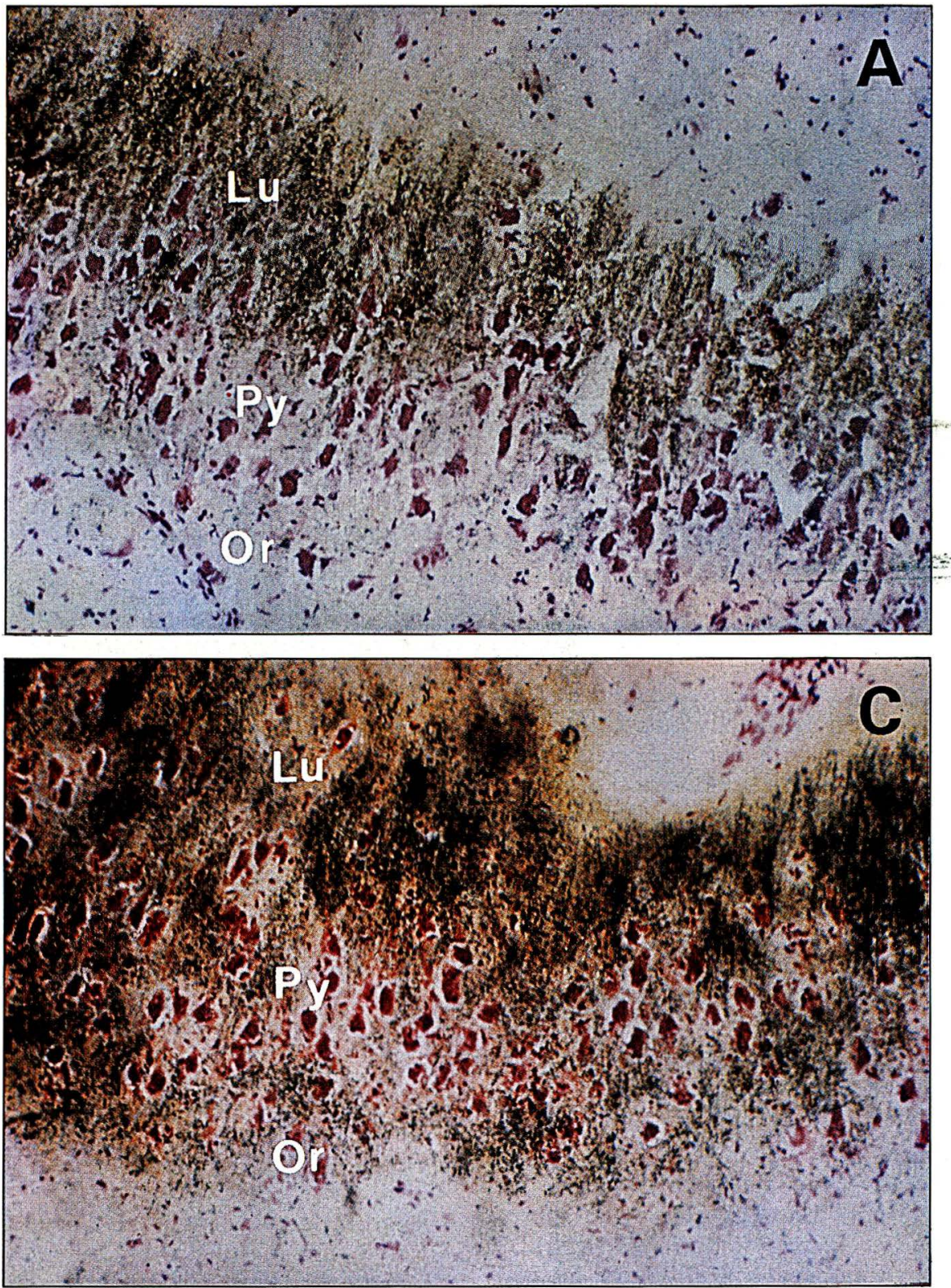

Figure 4. Bourgeonnement des fibres moussues chez l'enfant après épilepsie. $\boldsymbol{A}$ et $\boldsymbol{B}$ : cas témoins; $\boldsymbol{C}$ et $\boldsymbol{D}$ : cas épileptiques. La coloration de Timm (A et C) montre la présence des fibres moussues ectopiques (dans le stratum oriens) au niveau de la région CA3 chez des enfants épileptiques. Les images autoradiographiques des sites de liaison de haute affinité au KA ( $B$ et $D$ ) montrent que, chez le sujet épileptique, la densité des sites de liaison augmente significativement par rapport au sujet témoin de même áge dans la région CA3 

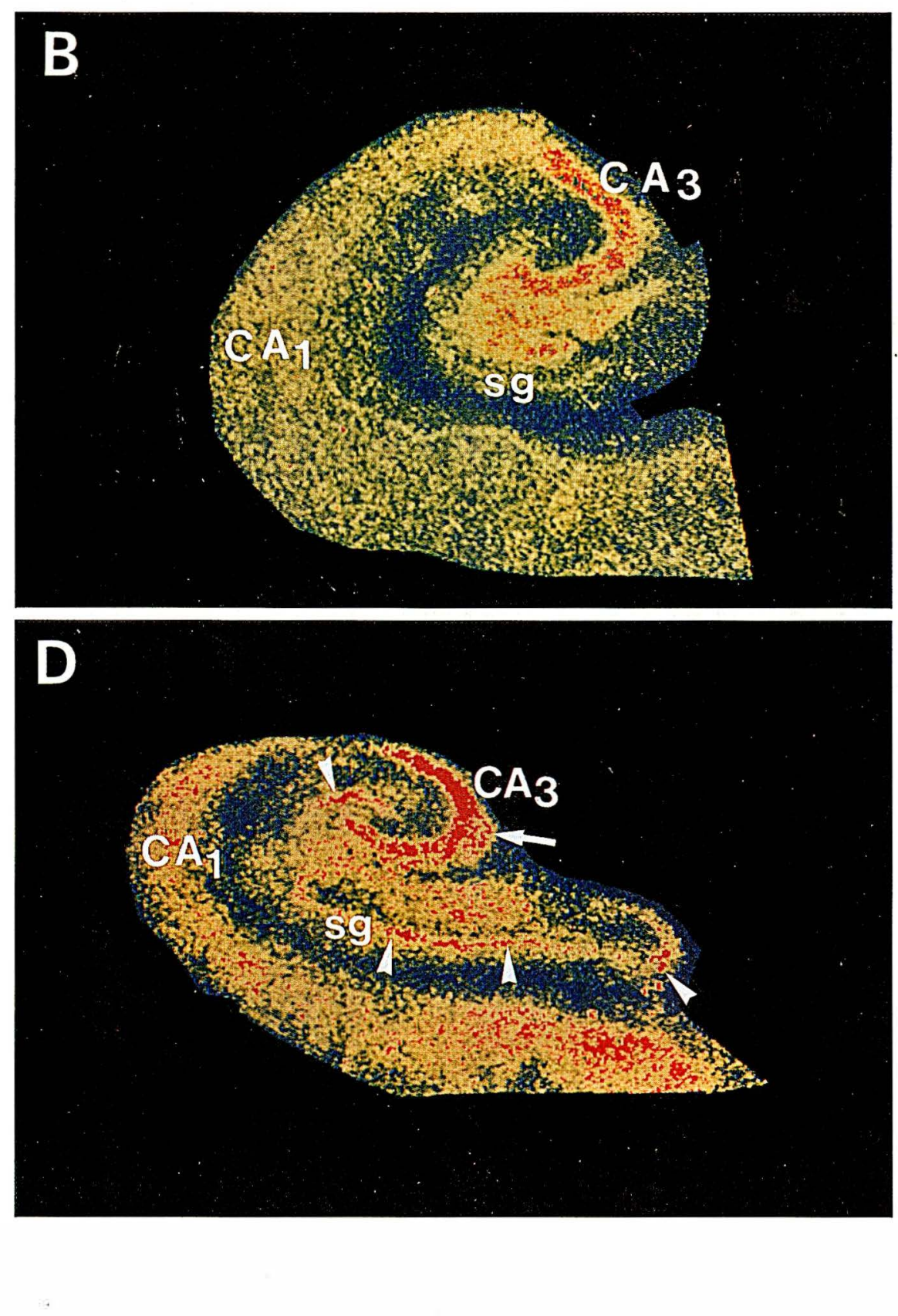

$\mathrm{Ca}^{2+}$ par le canal-récepteur NMDA. L'augmentation du calcium intracellulaire qui en résulte va déclencher, comme pour la potentialisation à long terme [22], une cascade d'événements qui vont se traduire par des modifications persistantes de l'efficacité synaptique :

a) Le kindling ou l'acide kaïnique [23-25] produisent une augmentation de l'expression du protooncogène $c$-fos au niveau de l'hippocampe et particulièrement de la fascia dentata. Fos est une protéine nucléaire, activée par l'augmentation du calcium intracellulaire, qui agit sur l'expression de certains gènes. Elle pourrait donc être impliquée dans la croissance neuritique et intervenir dans le bourgeonnement réactif des fibres moussues.

b) Les crises convulsives limbiques augmentent également la production de l'ARNm du NGF (nerve growth factor, protéine nécessaire au développement et au maintien des neurones sympathiques et sensoriels en culture - voir l'article de $\mathrm{P}$. Brachet dans ce numéro) au niveau des cellules granulaires de la fascia dentata [26] ; Funabashi et al. [27] ont d'ailleurs montré qu'un traitement intraventriculaire avec un antisérum dirigé contre le NGF retarde l'apparition du kindling amygdalien. Puisque le NGF semble avoir un effet direct dans le bourgeonnement axonique [28-30], il est tentant de suggérer que l'activité paroxystique augmente la libération de facteurs trophiques qui pourraient promouvoir le bourgeonnement des fibres moussues.

En conclusion, une brève activité paroxystique produit des effets quasi permanents au niveau de l'hippocampe avec la formation de nouvellestsynapses. Les facteurs et mécanismes impliqués pourraient être similaires à ceux responsables du remaniement synaptique observé après lésion du système nerveux [28] ou au cours du développement normal [29-30]

\section{$\rightarrow$}

(stratum lucidum-pyramidal) et dans la couche supragranulaire de la fascia dentata limages autoradiographiques traitées avec le système " autorad " ; IMS$T A R)$; les plus fortes densités de liaison ont été représentées en rouge, les intermédiaires en jaune et les plus faibles en vert et bleu). Lu, Py et Or: stratum lucidum, pyramidal et oriens de la région CA3, respectivement. sg: couche supragranulaire de la fascia dentata.

TIRÉS A PART

A. Represa. 


\section{RÉFÉRENCES}

26. Gall CM, Isackson PJ. Limbic seizures increase neuronal production of messenger RNA for nerve growth factor. Science 1989 ; 245 : 758-61.

27. Funabashi T, Sasaki H, Kimura F. Intraventricular injection of antiserum to nerve growth factor delays the development of amygdaloid kindling. Brain Res 1988 ; 458: $132-6$.

28. Springer JE, Loy R. Intrahippocampal injection of antiserum to nerve growth factor inhibits sympathohippocampal sprouting. Brain Res Bull 1985 ; 15 : 629-34.

29. Katz MJ. Quantitative effects of NGF on the growth of embryonic frog axons. Brain Res 1986 ; 366 : 211-6.

30. Gähwiler BH, Enz A, Hefti F. Nerve growth factor promotes development of the rat septo-hippocampal cholinergic projection in vitro. Neurosci Lett 1987 ; 75 : 6-10.

31. Ben-Ari Y, Represa A. Brief seizure episodes induce long-term potentiation and mossy fibre sprouting in the hippocampus. Trends Neurosci 1989 ; 13 : 312-8.

\section{Summary}

Synaptic reorganization of hippocampal mossy fibers in epilepsy

The mossy fibers of the hippocampus are a major intrahippocampal fiber system. They project from the granule cells of the fascia dentata to interneurons and CA3 pyramidal neurons. Mossy fibers have several unique features including high content of zinc in their terminals and high density of kainic acid binding sites. In experimental models of epilepsy (kindling and intracerebral administration of kainate) mossy fibers sprout and establish new connections, some of which are aberrant. Evidence in favor of similar sprouting has recently been found in human epilepsies. The aberrant mossy fibers and the associated increase in zinc and kainate binding sites may contribute to the hyperexcitability of the hippocampus in epilepsy. 